\title{
Can transperineal ultrasound improve the diagnosis of obstetric anal sphincter injuries?
}

\author{
Ka Wong ${ }^{1}$, Ranee Thakar ${ }^{2}$, Abdul Sultan ${ }^{2}$, and Vasanth Andrews ${ }^{3}$ \\ ${ }^{1}$ Croydon University Hospital \\ ${ }^{2}$ Croydon University Hospital \\ ${ }^{3}$ Lewisham Hospital NHS Trust
}

August 12, 2021

\begin{abstract}
Background: Women with missed Obstetric anal sphincter injuries (OASIs) are at an increased risk of anal incontinence. Objective: To assess the accuracy of 3D Transperineal Ultrasound (TPUS) compared with clinical examination for detecting OASIs. Design: Prospective Observational longitudinal cohort study. Setting: District General Hospital, UK. Population or sample: Women undergoing their first vaginal delivery immediately postpartum. Methods: Perineal trauma was initially assessed by accouchers and women were then re-examined by a trained research fellow. A 3D TPUS was performed immediately after delivery before suturing to look for OASIs. Main outcome measures: OASIs on clinical examination and on TPUS Main Results: Two hundred and sixty-four women participated and two hundred and twenty-six (86\%) delivered vaginally. Twenty-one (9\%) sustained OASIs. Six $(29 \%)$ of these tears were missed by the accoucher but were identified by the trained research fellow. TPUS identified 19 of the 21 (90.5\%) OASIs. One percent $(\mathrm{n}=2)$ had sonographic appearances of an anal sphincter defect and were not seen clinically. The positive and negative predictive of TPUS to detect OASIs were $91 \%$ and $99 \%$ respectively. TPUS identified $91 \%$ of OASIs compared to $71 \%$ detected by the accoucher. However, this was not statistically significant. Conclusions: More OASIs were identified on TPUS compared to examination. TPUS may have role in improving the detection rate of OASIs. Considering immense training and financial implications of using TPUS, attention needs to be focused on training to accurately identify anal sphincter defects on clinical examination. Funding:none Keywords: transperineal ultrasound imaging, obstetric anal sphincter injury
\end{abstract}

\section{Introduction}

Obstetric anal sphincter injury (OASI) is the most common cause of anal incontinence in women and can have a devastating effect on a woman's mental and physical health as well as their social wellbeing (1). In the United Kingdom, the rate of OASIs in primiparous women is $6.1 \%$. The rates have risen three-fold from 2000 to 2012 (2). A similar trend has also been observed in Nordic countries and Canada $(3,4)$.

It is recommended that all women should be examined by a midwife or doctor following a vaginal delivery to assess perineal trauma and anal sphincter disruption (5) by performing a digital rectal and vaginal examination to avoid missing OASI. It has been shown that when a trained clinician re-examines women after their initial assessment, that the detection rate of OASIs doubles (6).

Women with missed OASIs are more likely to develop anal incontinence $(7,8)$. Endoanal ultrasound (EAUS) has been evaluated to improve the detection of OASIs at delivery. Andrews et al (13) assessed the anal sphincter by performing an EAUS immediately postpartum. Women also had a clinical examination by the accoucher to assess perineal trauma and were re-examined by a trained clinical research fellow immediately after delivery. They found that $58 \%$ of OASIs were missed by doctors and midwives (13) and all of these were identified using EAUS. EAUS also had a false positive rate of $2 \%$ based on anal sphincter defects on 
scan that were not clinically identifiable. The feasibility of using EAUS immediately after delivery however is limited as it is expensive, requires training and is not readily available in most maternity units. EAUS is also known to cause more discomfort than other imaging modalities (9) including transperineal ultrasound (TPUS). Over the last decade there has been an interest in using TPUS to image the anal sphincter (912). The equipment is universally available and enables visualisation of the anal sphincter complex without disrupting anatomy. It also has the advantage of multiplanar imaging and three-dimensional (3D) volume storage which allows one to review images offline.

Although previous research highlights that OASIs can be missed by doctors and midwives $(6,13,14)$, many of these studies were performed more than 10 years ago. Training in both diagnosis and repair of OASIs has improved subsequently (15-17). The aim of this study was to establish whether 3D TPUS improves the detection of OASIs in women having their first vaginal delivery compared with clinical examination.

\section{Methods}

Women having their first vaginal delivery over a 16-month period between March 2016 and June 2017 at University Hospital Lewisham were invited to participate. Women were given a patient information leaflet about the study at 20-22 weeks gestation during anomaly scan appointment. Women were approached by a dedicated clinical research fellow in the maternity triage, antenatal ward and the labour ward in their early labour. During the initial contact, women were informed about the study and invited to participate. The inclusion criteria were a singleton pregnancy of 37 weeks of gestation or more, maternal age 18 years old or more, women having their first vaginal delivery, cephalic presentation, and being able to read and understand English.

All consenting women had their perineum examined after delivery by the accoucher. The clinical examination was conducted according to the individual's usual practice. All women then had their perineum re-examined by a trained clinical research fellow - this was carried out in a systematic fashion with adequate light and consisted of a rectal and vaginal examination. We minimised the measurement and technique variability by having all clinical examinations performed by a single trained research fellow who is experienced in diagnosing OASIs. All perineal tears were classified according to RCOG classification (18). When an OASI was identified by the clinical research fellow $(\mathrm{KW})$ that was missed by the doctor or midwife who conducted the initial examination it was verified by a senior registrar or consultant obstetrician, thereby minimising the risk of overdiagnosing OASIs by the clinical research fellow.

3D TPUS was performed at rest using a GE Voluson 730 system with a 4-8 MHz transabdominal curved array volume transducer after delivery and before suturing. Women were scanned by the trained clinical research fellow $(\mathrm{KW})$ in the supine position with knees semi-flexed after emptying their bladder. The transverse transducer placement allows the probe to keep a minimal distance from the anal sphincter and focus on the first two centimetres of the anal sphincter. The probe is then tilted to visualise the puborectalis muscle which appears as a ' $U$ ' shape. It is then angulated to obtain a transverse view of the anal canal and to visualise the whole length of the anal sphincter complex. A minimum of three ultrasound volumes are acquired and used for assessment of the external and internal anal sphincter. The 3D volume images are stored for offline assessment. Tomographic imaging (TUI) allows for evaluation of the entire EAS from the subcutaneous part of the EAS to its cranial termination. It is adjusted to have eight slices, with the interslice interval varying according to the individual sphincter length. Slice one corresponds with the puborectalis level, slice two the most cranial aspect of the EAS (deep level), slice three to six with the superficial level, and slice seven and eight the subcutaneous level. The EAS is seen in slices 2-8 and the IAS in slices 2-6. The angle of discontinuity of the ring shape of the EAS or IAS is captured with TPUS after delivery and prior to suturing and was later measured blindly. An EAS defect is diagnosed when three or more of the seven slices (between slice two and eight on TUI) showed a discontinuity of 30 degrees or more in the ring of muscle of the external sphincter (mixed echoic ring). Similarly IAS defects are diagnosed when two or more of the five slices (between slice two and slice six on TUI) show a discontinuity in the sonographic image of the internal sphincter (hypoechoic ring) (38). One independent investigator (KW) who was blinded to both information regarding the delivery and clinical assessments analysed the ultrasound images. Ten percent of the images 
were randomly selected and analysed by two independent investigators who were also blinded. Discrepancies were reviewed by a third blinded investigator.

\section{Statistical analysis}

A power calculation was performed. The calculation was based on the occurrence of OASIs being $6 \%$ in the first examination (clinical diagnosis of OASIs by midwives/doctors). As the data is 'paired' (i.e. two measurements, clinical examination and TPUS, for each subject), in addition to the OASIs rate for each method, it is also necessary to estimate the proportion of subjects were there will be a discordant response. This is where the outcome will be different from the two methods. With increased surveillance with TPUS and a detailed examination by a trained doctor, we predicted that the detection rate of OASIs would be rise from $6 \%$ to $12 \%$. Assuming that $10 \%$ of the subjects would have discordant responses, and also assuming a $5 \%$ significance level and $80 \%$ power, it was calculated that 216 women would be required for the study. An extra 12 patients were recruited as we anticipated that some of the ultrasound images would be in suboptimal quality to be included in the study. Sensitivity of a test was calculated by the number of true positives / the number or true positives plus number of false negatives. Specificity of a test was calculated by the number of true negatives / number of true negatives plus number of false positives. The positive predictive value of a test was calculated by the number positives of true positives / number of true positives plus number of false.

Data was entered onto a Microsoft Excel database and analysed by SPSS version 26 (Chicago, Illinois, USA). This study was approved by the South East Coast - Surrey Research Ethics Committee (REC 16/LO/2140).

\section{Results}

Two hundred and seventy-four women were invited, and 264 (95\%) women agreed to participate. Of the ten women (5\%) who declined to patriciate: six women did not provide any reason, one reported in pain, one was not interested and two declined because their partner did not want the women to participate. Two hundred and twenty-six women delivered vaginally. Two hundred and nineteen were nulliparous, six $(2.7 \%)$ had had a previous caesarean section and one $(0.5 \%)$ had had two previous caesarean sections (none of which were in the second stage of labour). One hundred and twenty-one (53.5\%) perineal tears were examined by midwives and $105(46.5 \%)$ by doctors (Table 1$)$. Twelve (4.6\%) women had an intact perineum, eight $(3 \%)$ a first-degree tear and $185(70.3 \%)$ a second degree tear. Twenty-one (9.3\%) women sustained OASIs: eight $(3 \%)$ a $3 \mathrm{a}$ tear and $13(4.9 \%)$ a $3 \mathrm{~b}$ tear. Of these, five women were examined by midwives who missed four (80\%) (Table 1) and sixteen were examined by doctors who missed two (12.5\%) OASIs (Table 2).

Two hundred and eighteen of the $226(96 \%)$ women had ultrasound images that were of a suitable quality for analysis. In $21(9.6 \%)$ women an anal sphincter defect was identified by TPUS. Table 3 shows the correlation between ultrasound and clinical findings. Of the 21 OASIs that occurred 19 (90.5\%) women had an anal sphincter defect identified with TPUS at delivery. No woman had an IAS injury that was diagnosed clinically or with TPUS (Table 3 ). In addition, there were two (1\%) defects seen on ultrasound that were not clinically detectable. The diagnostic performance of TPUS for detecting OASIs was evaluated, the positive and negative predictive of TPUS being $91 \%$ and $99 \%$ respectively (Table 4).

The relationship between the size of EAS defects identified clinically and by ultrasound was also analysed. There was no correlation between the grade of OASIs on clinical examination and on TPUS $\left(\chi^{2}\right.$ test, $\left.\mathrm{p}=0.14\right)$ (Table 5).

\section{Discussion}

\section{Main findings}

This is the first study to evaluate the anal sphincter immediately after delivery with TPUS. Nine percent of women sustained OASIs. TPUS identified $91 \%$ of OASIs compared to $71 \%$ detected by the accoucher suggesting that the detection rate with ultrasound may be better. In addition, there is poor correlation between the size of defects seen clinically when compared with TPUS. 


\section{Strengths and limitations}

The strengths of this study are that validated methods of ultrasound assessment were used to evaluate the anal sphincter complex. A power calculation was undertaken to establish the incidence of OASIs and an adequate sample size was achieved. Our study is the first to correlate clinical findings with TPUS in women undergoing their first vaginal delivery immediately postpartum. All TPUS scans were reviewed blindly without prior knowledge of clinical findings, thereby reducing the risk of detection bias.

The limitations of our study are that it was a single centre study, and most of the women were nulliparous, this decreases the prospect of generalisability. All the scans were performed by a single operator, which can potentially introduce significant bias; however, this factor also allowed for standardisation of the scans. It is acknowledged that the number of women who sustained OASIs is relatively small.

\section{Interpretation}

The rate of OASIS in primiparous women in the United Kingdom is 6.1\% (19), which was similar to that detected by the accoucher. However, when women were re-examined by the trained research fellow, the incidence of OASIs increased from $6 \%$ to $9 \%$. Previous studies have also shown that OASIs are missed by doctors and midwives $(6,13,14)$. Andrews et al performed a prospective study of 254 primiparous women immediately after delivery, and found that the prevalence of OASIs increased from $11 \%$ to $24.5 \%$ when women were re-examined by a trained clinical research fellow (13). Of these, $87 \%$ of OASIs were missed by midwives compared to $24 \%$ by doctors. Similarly, Groom et al independently re-assessed 121 women's perineum following a vaginal delivery and found that $40 \%$ of OASIs were being missed by accouchers (6). Both of these studies however are more than 15 year old and subsequently there has been an increase both in awareness and training in the diagnosis and management of OASIs (16). Hands on training courses in the management and diagnosis of OASIs are now mandatory for trainees in Obstetrics and Gynaecology the UK and have been shown to improve both the knowledge of perineal anatomy and techniques of identifying (15-17). Most missed OASIs however appear to be by midwives. In the UK there is no standardised assessment for the midwives in the diagnosis and repair of perineal tears. For obstetricians the Royal College of Obstetricians and Gynaecologists have developed objective structured assessment of surgical and technical skills for perineal tears. These are mandatory for obstetric trainees, and consideration should be given to implementing these for midwives as well.

This is the first study where TPUS was performed immediately after delivery and prior to suturing. $90.5 \%$ of anal sphincter defects seen clinically were confirmed on ultrasound and $99 \%$ of the clinically intact anal sphincters appeared to have an intact anal sphincter on 3D TPUS. We found that TPUS had a low positive predictive value to diagnose a sphincter defect and a high negative predictive value to detect an intact anal sphincter. This is in keeping with the findings of a cross-sectional study of 250 women who sustained OASIs that were scanned at $6-12$ weeks postpartum by TPUS, where they found that 3D TPUS had a low positive predictive value (0.37-0.63) and a high negative predictive value (0.85-0.95) when compared with EAUS. They found that 3D TPUS could identify an intact anal sphincter but only had a fair ability to diagnose an EAS and IAS defect (9). Although Taithongchai et al's (9) study is the most adequately powered study to date to compare the TPUS, TVUS with EAUS, it must be acknowledged that their training is of high standard and may not be generalisable.

TPUS had also been used to evaluate the anal sphincter complex during the early postpartum period. García-Mejido et al found that of the 146 women where TPUS was performed within 48 hours postpartum, 12 OASIs were detected by clinical examination and all such injuries were confirmed by TPUS (20). These results, however, should be interpreted with caution due to the small number of women who sustained OASIs and the study was not adequately powered.

In our study there were two anal sphincter defects (1\%) seen on ultrasound and classified as a second degree tear clinically. These may have been missed by the clinical research fellow. It may also represent a genuine "occult" sphincter injury. Genuine 'occult' anal sphincter injuries are defects that are only seen on ultrasound and not detected clinically (21). These injuries were previously believed to be "occult", but 
subsequently have been shown to represent clinically missed OASIs (13). Our findings were consistent with another observational study where $1 \%$ of OASI were seen on ultrasound but were not clinically detectable (13).

We found two OASIs that were diagnosed clinically but not seen on TPUS. The plausible explanation of the discrepancy between the clinical and ultrasound findings might be because of the technical difficulties performing an ultrasound examination immediately postpartum. It can be challenging due to oedema, haematoma and suture material (22) which may impair the image quality of the EAS. The IAS, however, seems to be easier to interpret due to its hypoechoic nature. In our study eight $(3.5 \%)$ ultrasound images were excluded due to quality of the images. This finding is consistent with another observational study by García-Mejido et al (23) who performed TPUS on women immediately postpartum and found that three anal sphincter defects $(2 \%)$ were not diagnosed on clinical examination but were detected in the subsequent ultrasound scan. They excluded five (3\%) images due to poor quality for analysis caused by the intense perineal oedema.

All OASIs in our study were classified according to the RCOG classification. Using this classification if less than $50 \%$ of the EAS is torn it is classified as a $3 \mathrm{a}$ tear, and as a $3 \mathrm{~b}$ tear when there is more than $50 \%$ involvement. We found that there is poor correlation between the size of an EAS defect seen clinically, when compared with ultrasound findings using TPUS similar to that seen on EAUS (13).

\section{Conclusion}

OASIs are still being missed by accouchers, and midwives failed to identify $80 \%$ of OASIS. TPUS identified $91 \%$ of OASIs compared to $71 \%$ detected by the accoucher suggesting that the detection rate with TPUS may be better. However, TPUS requires specialist skills to perform and interpret the images immediately after delivery and does not detect all OASIS and therefore cannot be a substitute for training in clinical diagnosis. More robust methods of education and assessment of competency therefore need to be implemented for midwives and doctors to improve the identification of OASIs.

\section{Declaration}

Financial Disclaimers/Conflict of Interest statement: None

Funding: none

\section{Author's participation in the manuscript:}

KW Wong: Protocol/project development, data collection and management, data analysis, manuscript writing

R Thakar: Protocol/project development, manuscript editing

AH Sultan: Protocol/project development, manuscript editing

V Andrews: Protocol/project development, data analysis, manuscript editing

\section{Acknowledgements}

We would like to thank P Bassett, Statistician from the Statsconsultancy Ltd, University of Reading for his help with the statistical analyses

\section{Ethical statement}

This study was approved by the South East Coast - Surrey Research Ethics Committee (REC 16/LO/2140).

\section{References}

1. Williams A, Lavender T, Richmond DH, Tincello DG. Women's experiences after a third-degree obstetric anal sphincter tear: a qualitative study. Birth. 2005;32(2):129-36. 
2. Gurol-Urganci I, Cromwell DA, Edozien LC, Mahmood TA, Adams EJ, Richmond DH, et al. Third- and fourth-degree perineal tears among primiparous women in England between 2000 and 2012: time trends and risk factors. BJOG. 2013;120(12):1516-25.

3. McLeod NL, Gilmour DT, Joseph KS, Farrell SA, Luther ER. Trends in major risk factors for anal sphincter lacerations: a 10-year study. J Obstet Gynaecol Can. 2003;25(7):586-93.

4. Laine K, Gissler M, Pirhonen J. Changing incidence of anal sphincter tears in four Nordic countries through the last decades. Eur J Obstet Gynecol Reprod Biol. 2009;146(1):71-5.

5. RCOG Greentop guideline 29: the management of third- and fourth-degree perineal tears: RCOG June 2015, www.rcog.org.uk.

6. Groom KM P-BS. Can we improve on the diagnosis of third degree tears? Eur J Obstet Gynecol Reprod Biol. 2002;101:19-21.

7. Taithongchai A, Veiga SI, Sultan AH, Thakar R. The consequences of undiagnosed obstetric anal sphincter injuries (OASIS) following vaginal delivery. Int Urogynecol J. 2020;31:635-41.

8. L R. Does a missed obstetric anal sphincter injury at time of delivery affect short-term functional outcome? Ann R Coll Surg Engl. 2018;100:26-32.

9. Taithongchai A, van Gruting IMA, Volloyhaug I, Arendsen LP, Sultan AH, R T. Comparing the diagnostic accuracy of 3 ultrasound modalities for diagnosing obstetric anal sphincter injuries. Am J Obstet Gynecol. 2019;221:134.

10. Garcia-Mejido JA, Gutierrez Palomino L, Fernandez Palacin A, Sainz-Bueno JA. [Applicability of 3/4D transperineal ultrasound for the diagnosis of anal sphincter injury during the immediate pospartum]. Cir Cir. 2017;85(1):80-6.

11. Shek KL, Zazzera VD, Atan IK, Rojas RG, Langer S, Dietz HP. The evolution of transperineal ultrasound findings of the external anal sphincter during the first years after childbirth. Int Urogynecol J. 2016;27(12):1899-903.

12. Roos AM, Abdool Z, Sultan AH, Thakar R. The diagnostic accuracy of endovaginal and transperineal ultrasound for detecting anal sphincter defects: The PREDICT study. Clin Radiol. 2011;66(7):597-604.

13. Andrews V, Sultan AH, Thakar R, PW J. Occult anal sphincter injuries-myth or reality? BJOG. 2006;113(2):195-200.

14. Sioutis D, Thakar R, Sultan AH. Overdiagnosis and rising rate of obstetric anal sphincter injuries (OASIS): time for reappraisal. Ultrasound Obstet Gynecol. 2017;50(5):642-7.

15. Ali-Masri H, Hassan S, Ismail K, Zimmo K, Zimmo M, Fosse E, et al. Enhancing recognition of obstetric anal sphincter injuries in six maternity units in Palestine: an interventional quality improvement study. BMJ Open. 2018;8(6):e020983.

16. Zimmo K, Laine K, Vikanes A, Fosse E, Zimmo M, Ali H, et al. Diagnosis and repair of perineal injuries: knowledge before and after expert training-a multicentre observational study among Palestinian physicians and midwives. BMJ Open. 2017;7(4):e014183.

17. Andrews V, Thakar R, Sultan AH. Structured hands-on training in repair of obstetric anal sphincter injuries (OASIS): an audit of clinical practice. Int Urogynecol J Pelvic Floor Dysfunct. 2009;20(2):193-9.

18. RCOG Greentop guideline 29: the management of third- and fourth-degree perineal tears: RCOG June 2015, www.rcog.org.uk

19. Thiagamoorthy G, Johnson A, Thakar R, Sultan AH. National survey of perineal trauma and its subsequent management in the United Kingdom. Int Urogynecol J. 2014;25(12):1621-7. 
20. García-Mejido JA, Palomino LG, Palacín AF, Sainz-Bueno JA. Applicability of 3/4D transperineal ultrasound for the diagnosis of anal sphincter injury during the immediate postpartum. Cirugía y Cirujanos (English Edition). 2017;85(1):80-6.

21. Sultan AH KM, Hudson CN, Thomas JM, Bartram CI. Anal sphincter disruption during vaginal delivery N Engl J Med. 1993;329:1905-11.

22. Faltin DL, Boulvain M, Stan C, Epiney M, Weil A, O I. Intraobserver and interobserver agreement in the diagnosis of anal sphincter tears by postpartum endosonography. Ultrasound Obstet Gynecol. 2003;21(4):3757 .

23. García-Mejido JA, Palomino LG PA, JA S-B. Applicability of 3/4D transperineal ultrasound for the diagnosis of anal sphincter injury during the immediate postpartum. Cirugía y Cirujanos (English Edition). 2017;85(1):80-6.

Table 1 Perineal trauma diagnosed by midwives and research fellow

\begin{tabular}{|c|c|c|c|}
\hline $\begin{array}{l}\text { Research fellow } \\
\text { diagnosis }(\%) \\
\mathrm{n}=121\end{array}$ & $\begin{array}{l}\text { Research fellow } \\
\text { diagnosis }(\%) \\
\mathrm{n}=121\end{array}$ & $\begin{array}{l}\text { Midwives' diagnosis } \\
(\%) \\
\mathrm{n}=121\end{array}$ & $\begin{array}{l}\text { Agreement } \\
(\%)\end{array}$ \\
\hline Intact perineum & $12(9.9)$ & Intact perineum $12(9.9)$ & 100 \\
\hline First degree tear & $6(5)$ & First degree tear $6(5)$ & 100 \\
\hline Second degree tear & $98(81)$ & $\begin{array}{l}\text { Second degree tear } 102 \\
(84.3)\end{array}$ & 96 \\
\hline Third degree tear & $5(4.1)$ & $\begin{array}{l}\text { Second degree tear } 4 \\
\text { (3.3) Third degree tear } 1 \\
(0.8)\end{array}$ & 20 \\
\hline
\end{tabular}

Table 2 Perineal trauma diagnosis by doctors and research fellow

\begin{tabular}{llll}
\hline Doctors diagnosis (\%) $\mathrm{n}=105$ & Doctors diagnosis (\%) $\mathrm{n}=105$ & $\begin{array}{l}\text { Research Fellow diagnosis }(\%) \\
\mathrm{n}=105\end{array}$ & $\begin{array}{l}\text { Research Fellow diagnosis } \\
\mathrm{n}=105\end{array}$ \\
\hline Intact perineum & 0 & $\begin{array}{l}\text { Intact perineum } \\
0\end{array}$ & $\begin{array}{l}\text { Intact perineum } \\
0\end{array}$ \\
First degree tear & $2(1.9)$ & $\begin{array}{l}\text { First degree tear } \\
2(1.9)\end{array}$ & $\begin{array}{l}\text { First degree tear } \\
2(1.9)\end{array}$ \\
Second degree tear & $89(84.8)$ & $\begin{array}{l}\text { Second degree tear } \\
\text { Second degree tear }\end{array}$ & $87(82.9)$ \\
Third degree tear & $16(15.2)$ & $16(15.2)$ & $\begin{array}{l}\text { Third degree tear } \\
14(13.3)\end{array}$ \\
\hline
\end{tabular}

Table 3 Transperineal ultrasound scan findings

\begin{tabular}{|c|c|c|c|c|}
\hline Type of injury & Type of injury & $\begin{array}{l}\text { Clinical diagnosis } \mathrm{n} \\
=226(\%)\end{array}$ & $\begin{array}{l}\text { TPUS anal } \\
\text { sphincter defects } \\
\text { prior to perineal } \\
\text { repair } \mathrm{n}=218^{*}\end{array}$ & Agreement (\%) \\
\hline Intact perineum & Intact perineum & $12(4.6)$ & $0 / 12$ & 100 \\
\hline Frist degree tear & Frist degree tear & $8(3)$ & $0 / 8$ & 100 \\
\hline
\end{tabular}




\begin{tabular}{|c|c|c|c|c|}
\hline Type of injury & Type of injury & $\begin{array}{l}\text { Clinical diagnosis } \mathrm{n} \\
=226(\%)\end{array}$ & $\begin{array}{l}\text { TPUS anal } \\
\text { sphincter defects } \\
\text { prior to perineal } \\
\text { repair } \mathrm{n}=218^{*}\end{array}$ & Agreement (\%) \\
\hline $\begin{array}{l}\text { Second degree } \\
\text { tear }\end{array}$ & $\begin{array}{l}\text { Second degree } \\
\text { tear }\end{array}$ & $185(70.3)$ & $2 / 177(1)$ & 99 \\
\hline \multirow[t]{4}{*}{$\begin{array}{l}\text { All third/fourth } \\
\text { degree tears }\end{array}$} & $\begin{array}{l}\text { All third/fourth } \\
\text { degree tears }\end{array}$ & $21(7.9)$ & $19 / 21(90.5)$ & 90.5 \\
\hline & 3a tear & $8(3)$ & $7 / 8(87.5)$ & 87.5 \\
\hline & 3b tear & $13(4.9)$ & $12 / 13(92.3)$ & 92.3 \\
\hline & $3 \mathrm{c} / 4^{\text {th }}$ degree tear & 0 & 0 & 100 \\
\hline
\end{tabular}

*Eight ultrasound images were excluded due to quality of images

Table 4 Diagnostic performance of TPUS for the detection of OASIs

\begin{tabular}{lll}
\hline Statistic & $\mathrm{n} / \mathrm{N}$ & Estimate $(95 \% \mathrm{CI})$ \\
\hline Sensitivity & $19 / 21$ & $91 \%(70 \%, 99 \%)$ \\
Specificity & $195 / 197$ & $99 \%(96 \%, 100 \%)$ \\
Positive Predictive Value & $19 / 21$ & $91 \%(70 \%, 99 \%)$ \\
Negative Predictive Value & $195 / 197$ & $99 \%(96 \%, 100 \%)$ \\
\hline
\end{tabular}

Table 5 Correlation between complete and partial tears of the external anal sphincter on ultrasound and clinical findings

\begin{tabular}{llll}
\hline Classification of OASIs & $2^{\text {nd }} \mathrm{n}=2$ & 3 a tear $\mathrm{n}=8$ & $3 \mathrm{~b}$ tear $\mathrm{n}=13$ \\
\hline $\begin{array}{l}\text { No defect on ultrasound } \\
\mathrm{n}=2\end{array}$ & 0 & 1 & 1 \\
$\begin{array}{l}\text { Partial defect on } \\
\text { ultrasound } \mathrm{n}=8\end{array}$ & 0 & 3 & 5 \\
$\begin{array}{l}\text { Complete defect on } \\
\text { ultrasound } \mathrm{n}=13\end{array}$ & 2 & 4 & 7 \\
\hline
\end{tabular}

